\title{
钯催化咖啡因 $\mathrm{C}$ - $\mathrm{H}$ 键直接烷氧基羰基化反应生成 8-酯基咖啡因衍生物
}

\author{
苏吕 ${ }^{a}$ 肖含兵 ${ }^{a}$ 苑雨萌 ${ }^{a}$ 张晓凤 ${ }^{a}$ 林 深 ${ }^{a}$ 黄秋锋*,,$b$ \\ ( ${ }^{a}$ 福建师范大学化学与化工学院 福州 350007) \\ ( ${ }^{b}$ 福建省高分子材料重点实验室 福州 350007)
}

\begin{abstract}
摘要 咖啡因羰基衍生物在药物活性及生物荧光方面表现优异性能, 其简便合成方法备受关注. 从咖啡因 $\mathrm{C}-\mathrm{H}$ 键直 接出发, 研究了过渡金属钯配合物催化咖啡因 $\mathrm{C}-\mathrm{H}$ 键与醇的氧化羰基化反应. 以 $\mathrm{PdCl}_{2}\left(\mathrm{PPh}_{3}\right)_{2}$ 为催化剂, 以醋酸铜为 氧化剂的反应体系下, 不同咖啡因衍生物与不同醇均能进行氧化羰基化反应，得到了一系列 8-酯基咖啡因衍生物. 反 应所用 $\mathrm{CO}$ 为一个大气压，操作简便，溴、烯烃和苯环等官能团都与体系有很好的兼容性.
\end{abstract}

关键词 钯; $\mathrm{C}-\mathrm{H}$ 键活化; 羊基化; 咖啡因; 一氧化碳

\section{Palladium-Catalyzed C-H Alkoxycarbonylation of Caffeines: Synthesis of 8-Ester-substituted Caffeines}

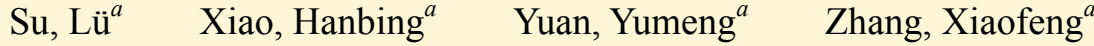 \\ Lin, Shen ${ }^{a} \quad$ Huang, Qiufeng*,a,b \\ ( ${ }^{a}$ College of Chemistry \& Chemical Engineering, Fujian Normal University, Fuzhou 350007) \\ ( ${ }^{b}$ Fujian Key Laboratory of Polymer Materials, Fuzhou 350007)
}

\begin{abstract}
Carbonyl-substituted caffeine derivatives have attracted much attention due to their potent pharmaceutical activity and interesting fluorescent properties. An efficient synthesis of 8-ester-substituted caffeines through palladium-catalyzed $\mathrm{C}-\mathrm{H}$ alkoxycarbonylation was developed. The reaction was carried out in the presence of $\mathrm{PdCl}_{2}\left(\mathrm{PPh}_{3}\right)_{2}$ and $\mathrm{Cu}(\mathrm{OAc})_{2}$ under 101 $\mathrm{kPa} \mathrm{CO}$ atmosphere in 1,4-dioxane, providing diversified 8-ester-substituted caffeines in reasonable to good yields. The approach was characterized by using atmospheric pressure of carbon monoxide and broad functional group tolerance.

Keywords palladium; C-H bond acitivation; carbonylation; caffeine; carbon monoxide
\end{abstract}

过渡金属催化羰基化反应是构建 $\mathrm{C}-\mathrm{C}$ 键的重要手 段之一, 同时羰基化反应可以在有机分子中引入重要官 能团羰基, 成为有机合成化学研究的前沿领域之一. 一 氧化碳 $(\mathrm{CO})$ 是一种常见的 $\mathrm{C} 1$ 化学资源, 对 $\mathrm{CO}$ 催化活 化的羰基化反应研究有显著的经济效益及环境效益; 另 一方面, $\mathrm{CO}$ 作为碳基源的羰基化反应一一插羰反应大 多数属于高原子经济性反应, 符合现代绿色化学发展的 要求. 所以以 $\mathrm{CO}$ 为羰基源的羰基化反应一直以来都是 $\mathrm{C} 1$ 资源高值化利用的重要研究方向及热点 ${ }^{[1]}$. 自从 Heck 首次报道以 $\mathrm{C}-\mathrm{X}(\mathrm{X}=$ 卤素或拟卤素)键出发的过
渡金属催化的羰基化反应之后 ${ }^{[2]}$, 由 $\mathrm{C}-\mathrm{X}$ 化合物出发 的羰基化反应取得了重大的进展 ${ }^{[3]}$. 但是 $\mathrm{C}-\mathrm{X}$ 化合物 制备成本高, 反应后会生成的 $\mathrm{HX}$ 酸副产物，原子利用 率低且对环境不友好. 近年来, 直接从 $\mathrm{C}-\mathrm{H}$ 键出发的 羰基化反应受到更多化学工作者的重视 ${ }^{[4]}$.

8-取代咖啡因衍生物是一类重要的有机分子, 在医 药方面表现出良好的药理性质 ${ }^{[5]}$, 如具有 $\beta$-二酮酸 (DKA)药效基团的 8-取代咖啡因衍生物分子(图 1)具有 抑制 HIV-1 整合酶活性的分子, 成为抗 HIV 药物 ${ }^{[6]}$; 同 时 8-取代咖啡因衍生物在生物苂光方面也表现出优异

* Corresponding author. E-mail: qiufenghuang@fjnu.edu.cn

Received October 17, 2016; revised December 8, 2016; published online December 12, 2016.

Project supported by the National Natural Science Foundation of China (Nos. 2110322, 6152010615) and the Foundation of Fujian Educational Committee (No. JK2014010).

国家自然科学基金(Nos. 2110322, 6152010615)和福建省教育厅 K 类基金(No. JK2014010)资助项目. 
的潜能. 尽管科学家们已经发现了许多发光探针如生物 发光蛋白、无机纳米颗粒、有机染料、稀土配合物等都 可用于生物成像 ${ }^{[7]}$, 但咖啡因这类体积小的苂光标记分 子具有低毒性和优良的生物相容性, 是生物成像方面重 要的研究方向之一 ${ }^{[8]}$. 本文将直接从咖啡因 $\mathrm{C}-\mathrm{H}$ 键出 发, 在 $\mathrm{CO}$ 气氛中, 以咖啡因与醇的羰基化反应作为研 究对象, 探索出 8-酯基取代咖啡因衍生物的新型简便合 成方法. 目前合成 8-酯基取代咖啡因衍生物的方法是从 咖啡因的卤代物出发得到目标产物, 此类合成路线缺点 明显, 如路线繁琐、底物范围狭窄及对环境不友好 ${ }^{[9]}$.

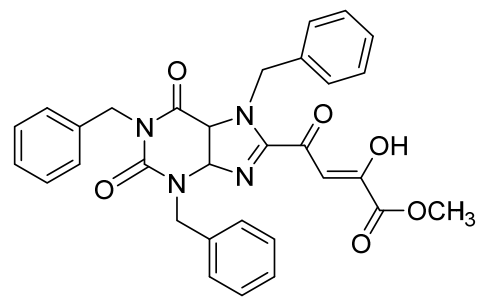

图 1 具有生物活性的 8-取代咖啡因衍生物

Figure 1 8-Substituted caffeine derivative with biological activities

\section{1 结果与讨论}

以咖啡因(1a)与正丁醇的氧化羰基化反应为模板反 应, 以 1,4-二氧六环为溶剂, $\mathrm{CO}$ 压力为一个大气压, 反 应温度 $120{ }^{\circ} \mathrm{C}$ 下对催化剂、氧化剂和碱进行了篮选. 过 渡金属催化 $\mathrm{C}-\mathrm{H}$ 活化并官能团反应中, 钯盐是最常见 的催化剂 ${ }^{[10]}$, 首先用醋酸钯为催化剂对反应进行了多 种尝试, 包括氧化剂、碱及各种添加剂的考察, 但反应 都不能进行(表 1, Entry 1). $\mathrm{Pd}(\mathrm{OPiv})_{2} 、 \operatorname{Pd}(\mathrm{TFA})_{2}$ 以及 $\mathrm{PdCl}_{2}$ 同样也没有催化活性(表 1, Entries 2 4). 我们还 尝试了其它过渡金属盐或配合物如 $\left[\mathrm{RuCl}_{2}(\mathrm{cod})\right]_{n} 、 \mathrm{RhCl}_{3}$ 和 $\left[\mathrm{Cp}^{*} \mathrm{RhCl}_{2}\right]_{2}$, 反应也基本不发生(表 1, Entries 5 7). 添加 $\mathrm{PPh}_{3}$ 配体对反应有很大地促进作用, 使用 $\mathrm{PdCl}_{2} / \mathrm{PPh}_{3} / \mathrm{Cu}(\mathrm{OAc})_{2}$ 催化剂体系时, 目标产物产率为 $30 \%$ (表 1, Entry 8), 将催化剂换成 $\mathrm{PdCl}_{2}\left(\mathrm{PPh}_{3}\right)_{2}$ 后, 可以 高分离产率得到目标产物( $83.0 \%$; 表 1, Entry 9). 接着 以 $\mathrm{PdCl}_{2}\left(\mathrm{PPh}_{3}\right)_{2}$ 为催化剂考察了几种常见的氧化剂发现 醋酸铜 $\mathrm{Cu}(\mathrm{OAc})_{2}$ 表现出较好的活性(表 1, Entries 10 13). 过渡金属催化 $\mathrm{C}-\mathrm{H}$ 活化反应中, 碱通常可以作为 酸的中和剂, 加速 $\mathrm{C}-\mathrm{H}$ 键的断裂 ${ }^{[11]}$, 因此我们对几种 碱进行了简单的考察(表 1 , Entries 14 16). 结果表明碱 的添加对反应没有帮助, 甚至是有害的. 比如添加 DBACO 后反应产率降低至 39\%(表 1, Entry 14), 而添加 $\mathrm{K}_{2} \mathrm{CO}_{3}$ 则使反应不能进行(表 1 , Entry 15). 减少催化剂 $\mathrm{PdCl}_{2}\left(\mathrm{PPh}_{3}\right)_{2}$ 的用量及氧化剂 $\mathrm{Cu}(\mathrm{OAc})_{2}$ 的用量, 都导致 反应产率大幅度下降(表 1 , Entries 17～18).
表 1 反应条件优化 ${ }^{a}$

Table 1 Optimization of the reaction conditions

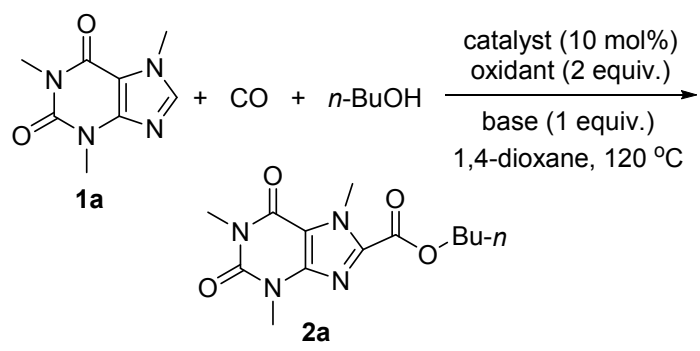

\begin{tabular}{cllcc}
\hline Entry & \multicolumn{1}{c}{ Catalyst } & \multicolumn{1}{c}{ Oxidant } & Base & Yield $^{b} \%$ \\
\hline 1 & $\mathrm{Pd}(\mathrm{OAc})_{2}$ & $\mathrm{Cu}(\mathrm{OAc})_{2}$ & & 0 \\
2 & $\mathrm{Pd}(\mathrm{OPiv})_{2}$ & $\mathrm{Cu}(\mathrm{OAc})_{2}$ & & 0 \\
3 & $\mathrm{Pd}^{2}(\mathrm{TFA})_{2}$ & $\mathrm{Cu}(\mathrm{OAc})_{2}$ & & 0 \\
4 & $\mathrm{PdCl}_{2}$ & $\mathrm{Cu}(\mathrm{OAc})_{2}$ & & 0 \\
5 & {$\left[\mathrm{RuCl}_{2}(\mathrm{cod})\right]_{n}$} & $\mathrm{Cu}(\mathrm{OAc})_{2}$ & & 0 \\
6 & $\mathrm{RhCl}_{3}$ & $\mathrm{Cu}(\mathrm{OAc})_{2}$ & & 0 \\
7 & {$\left[\mathrm{Cp}^{*} \mathrm{RhCl}_{2}\right]_{2}$} & $\mathrm{Cu}(\mathrm{OAc})_{2}$ & & 0 \\
$8^{c}$ & $\mathrm{PdCl}_{2} / \mathrm{PPh}_{3}$ & $\mathrm{Cu}(\mathrm{OAc})_{2}$ & & 30.0 \\
9 & $\mathrm{PdCl}_{2}\left(\mathrm{PPh}_{3}\right)_{2}$ & $\mathrm{Cu}(\mathrm{OAc})_{2}$ & & 83.0 \\
10 & $\mathrm{PdCl}_{2}\left(\mathrm{PPh}_{3}\right)_{2}$ & $\mathrm{AgOAc}$ & & 0 \\
11 & $\mathrm{PdCl}_{2}\left(\mathrm{PPh}_{3}\right)_{2}$ & $\mathrm{Ag} \mathrm{CO}_{3}$ & & Trace \\
12 & $\mathrm{PdCl}_{2}\left(\mathrm{PPh}_{3}\right)_{2}$ & $\mathrm{Oxone}$ & & 0 \\
13 & $\mathrm{PdCl}_{2}\left(\mathrm{PPh}_{3}\right)_{2}$ & $\mathrm{Benzoquinone}$ & & Trace \\
14 & $\mathrm{PdCl}_{2}\left(\mathrm{PPh}_{3}\right)_{2}$ & $\mathrm{Cu}(\mathrm{OAc})_{2}$ & $\mathrm{DABCO}_{2}$ & 39.0 \\
15 & $\mathrm{PdCl}_{2}\left(\mathrm{PPh}_{3}\right)_{2}$ & $\mathrm{Cu}(\mathrm{OAc})_{2}$ & $\mathrm{~K}_{2} \mathrm{CO}_{3}$ & Trace \\
16 & $\mathrm{PdCl}_{2}\left(\mathrm{PPh}_{3}\right)_{2}$ & $\mathrm{Cu}(\mathrm{OAc})_{2}$ & $\mathrm{Et}_{3} \mathrm{~N}$ & 18.9 \\
$17^{d}$ & $\mathrm{PdCl}_{2}\left(\mathrm{PPh}_{3}\right)_{2}$ & $\mathrm{Cu}(\mathrm{OAc})_{2}$ & & 26.3 \\
$18^{e}$ & $\mathrm{PdCl}_{2}\left(\mathrm{PPh}_{3}\right)_{2}$ & $\mathrm{Cu}(\mathrm{OAc})_{2}$ & & 15.7 \\
\hline
\end{tabular}

${ }^{a}$ Reaction conditions: caffeine (1a, $\left.0.2 \mathrm{mmol}\right), n-\mathrm{BuOH}(0.3 \mathrm{~mL})$, catalyst $(10$ mol\%), oxidant (2 equiv.), base (1 equiv.), $\mathrm{CO}(101 \mathrm{kPa})$ in 1,4-dioxane (1 $\mathrm{mL})$ at $120{ }^{\circ} \mathrm{C}$ for $24 \mathrm{~h} .{ }^{b}$ Isolated yield. ${ }^{c} \mathrm{PPh}_{3}(20 \mathrm{~mol} \%) .{ }^{d} \mathrm{PdCl}_{2}\left(\mathrm{PPh}_{3}\right)_{2}(5$ $\mathrm{mol} \%) .{ }^{e} \mathrm{Cu}(\mathrm{OAc})_{2}$ (1 equiv.)

在以 $\mathrm{PdCl}_{2}\left(\mathrm{PPh}_{3}\right)_{2}$ 为催化剂, $\mathrm{Cu}(\mathrm{OAc})_{2}$ 为氧化剂的 反应条件下，我们对反应溶剂对反应的影响进行了考 察, 结果列于表 2. 从表可以看出在极性溶剂 $N, N$-二甲 基甲酰胺(DMF)、二甲基亚砜(DMSO)和 $N, N$-二甲基乙 酰胺(DMA)中反应很难进行, 其中 DMSO 为溶剂时产 率只有 $9.0 \%$, DMF 和 DMA 为溶剂时反应都不能进行, 反应原料基本回收; 非极性溶剂作为反应溶剂时反应产 率有明显改善, 其中 1,4-二氧六环中目标产物产率最高.

综合以上反应条件结果, $\mathrm{PdCl}_{2}\left(\mathrm{PPh}_{3}\right)_{2}$ 为催化剂对 咖啡因与丁醇的羰基化反应的最佳反应条件是：10 mol\% $\mathrm{PdCl}_{2}\left(\mathrm{PPh}_{3}\right)_{2}, 2$ equiv. $\mathrm{Cu}(\mathrm{OAc})_{2}$, 咖啡因与丁醇的 物质的量比为 $1: 16.4$, 溶剂为 $1,4-$ 二氧六环, 反应温度 $120{ }^{\circ} \mathrm{C}, \mathrm{CO}$ 压力为 $101 \mathrm{kPa}$ ，反应时间 $24 \mathrm{~h}$.

上述优化反应条件基础上，首先对过渡金属催化的 一系列咖啡因衍生物与正丁醇的氧化羰基化反应进行 了考察(图 2). 1 位烷基取代咖啡因(2b $\sim 2 \mathbf{e}$ )和 7 位烷基 取代的咖啡因 $(\mathbf{2 f} \sim \mathbf{2 h})$ 可以很好地进行烷氧基羰基化反 
表 2 反应溶剂筛选 ${ }^{a}$

Table 2 Screening of the solvents

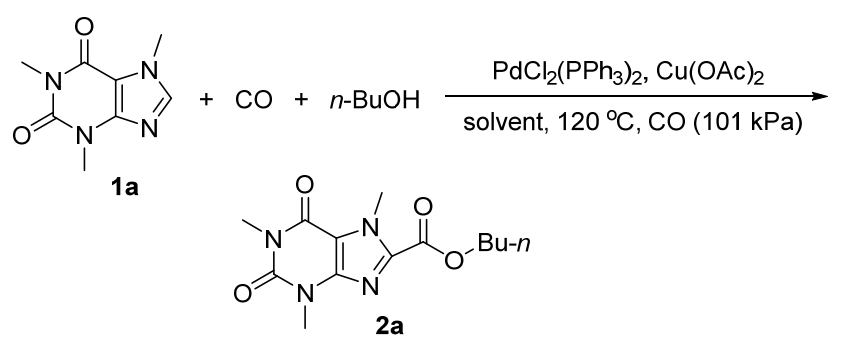

\begin{tabular}{clc}
\hline Entry & Solvent & Yield $^{b} / \%$ \\
\hline 1 & 1,4-Dioxane & 83.0 \\
2 & Toluene & 34.6 \\
3 & Anisole & 18.2 \\
4 & DMF & 0 \\
5 & DMSO & 9.0 \\
6 & DMA & 0 \\
\hline
\end{tabular}

${ }^{a}$ Reaction conditions: caffeine $(0.2 \mathrm{mmol}), \mathrm{n}-\mathrm{BuOH}(0.3 \mathrm{~mL})$, catalyst $(10$ mol\%), $\mathrm{Cu}(\mathrm{OAc})_{2}$ (2 equiv.), $\mathrm{CO}(1 \mathrm{~atm})$ in solvent $(1 \mathrm{~mL})$ at $120{ }^{\circ} \mathrm{C}$ for 24 h. ${ }^{b}$ Isolated yield.

应, 均以较高产率得到相应的咖啡因的酯化产物, 唯一 例外的是 7-烯丙基咖啡因, 目标产物产率仅 29.8\% (2i), 可能的原因是过渡金属钯配合物可以与靠近反应中心 (8 位 $\mathrm{C}-\mathrm{H}$ 键)的烯丙基配位从而影响其催化羰基化反<smiles>CCCCOC(=O)c1nc2c(c(=O)n(C)c(=O)n2C)n1CC</smiles>

2b, $74.8 \%$<smiles>CCCCOC(=O)c1nc2c(c(=O)n(CC)c(=O)n2C)n1C</smiles>

2f, $52.2 \%$<smiles>C=CCn1c(C(=O)OCCCC)nc2c1c(=O)n(C)c(=O)n2C</smiles>

$2 \mathrm{i}, 29.8 \%$<smiles>CCOC(=O)c1nc2c(c(=O)n(C)c(=O)n2C)n1C</smiles>

$2 \mathrm{~m}, 70.4 \%$<smiles>CCCCOC(=O)c1nc2c(c(=O)n(C)c(=O)n2C)n1CCC</smiles>

2c, $70.6 \%$<smiles>CCCCOC(=O)c1nc2c(c(=O)n(CCC)c(=O)n2C)n1C</smiles>

$2 g, 96.2 \%$<smiles>C=CCCOC(=O)c1nc2c(c(=O)n(C)c(=O)n2C)n1C</smiles>

2j, $43.2 \%$

应活性，而没有靠近反应中心的烯丙基则可以很好地在 反应体系中兼容(2h). 从图中还可以看出 7-位取代基的 位阻对反应活性影响不大，乙基、丙基甚至茮基取代咖 啡因都可以较好地参与反应，分别获得 $74.8 \%(2 \mathrm{~b})$ 、 $70.6 \%(2 \mathrm{c})$ 和 $95.9 \%(2 \mathrm{e})$ 的产率. 需要指出的是 $\mathrm{Br}$ 官能团 在反应中不受影响, 可以为目标产物的进一步衍生化提 供可能(2d).

在考察了咖啡因的底物范围后, 研究了醇的底物适 用范围. 伯醇如甲醇、乙醇和茮醇都能与咖啡因反应得 到较好产率的咖啡因酯化产物. $m$-甲氧基茮醇以及 3 -丁 烯-1-醇在标准条件下反应产率不高，分别为 42.4\% (2p) 和 43.2\% (2j). 仲醇如异丙醇也能够与咖啡因进行羰基 化反应, 但产率偏低 $(31.2 \%, 2 \mathrm{n})$, 而叔醇不能够与咖啡 因进行反应, 原因可能是空间位阻效应(20). 由于苯酚 中羟基的亲核性比较弱，在反应体系中不能作为反应物 与咖啡因进行羰基化反应.

基于实验结果与文献报道 ${ }^{[13]}$, 以咖啡因与醇的羰 基化反应为例子, 我们对反应机理进行了假设. 如 Scheme 1 所示, 首先咖啡因与二价钯物种进行亲电钯化 反应得到中间体 $\mathbf{A}$ ，接着一氧化碳插入得到中间体 $\mathbf{B}$, 随后醇作为亲核试进攻钯中心并脱去一分子 $\mathrm{HX}$ 生成中 间体 $\mathbf{C}$, 亲核性较弱的高位阻醇如叔醇会影响其反应活<smiles>CCCCOC(=O)c1nc2c(c(=O)n(C)c(=O)n2C)n1CCCBr</smiles>

2d, $73.4 \%$<smiles>CCCCOC(=O)c1nc2c(c(=O)n(C)c(=O)n2C)n1Cc1ccccc1</smiles>

2e, $95.9 \%$<smiles>C=CCn1c(=O)c2c(nc(C(=O)OCCCC)n2C)n(C)c1=O</smiles>

2h, $74.2 \%$<smiles>Cn1c(=O)c2c(nc(C(=O)OCc3ccccc3)n2C)n(C)c1=O</smiles>

2k, $70.2 \%$<smiles>COC(=O)c1nc2c(c(=O)n(C)c(=O)n2C)n1C</smiles>

2I, $68.2 \%$<smiles>CC(C)OC(=O)c1nc2c(c(=O)n(C)c(=O)n2C)n1C</smiles>

2n, $31.2 \%$<smiles>CCC(C)(C)OC(=O)c1nc2c(c(=O)n(C)c(=O)n2C)n1C</smiles>

2o, $0 \%$<smiles>COc1cccc(COC(=O)c2nc3c(c(=O)n(C)c(=O)n3C)n2C)c1</smiles>

$2 p, 42.4 \%$

图 2 钯催化咖啡因 $\mathrm{C}-\mathrm{H}$ 直接羰基化反应

Figure 2 Palladium-catalyzed $\mathrm{C}-\mathrm{H}$ alkoxylcarbonylation of caffeines 


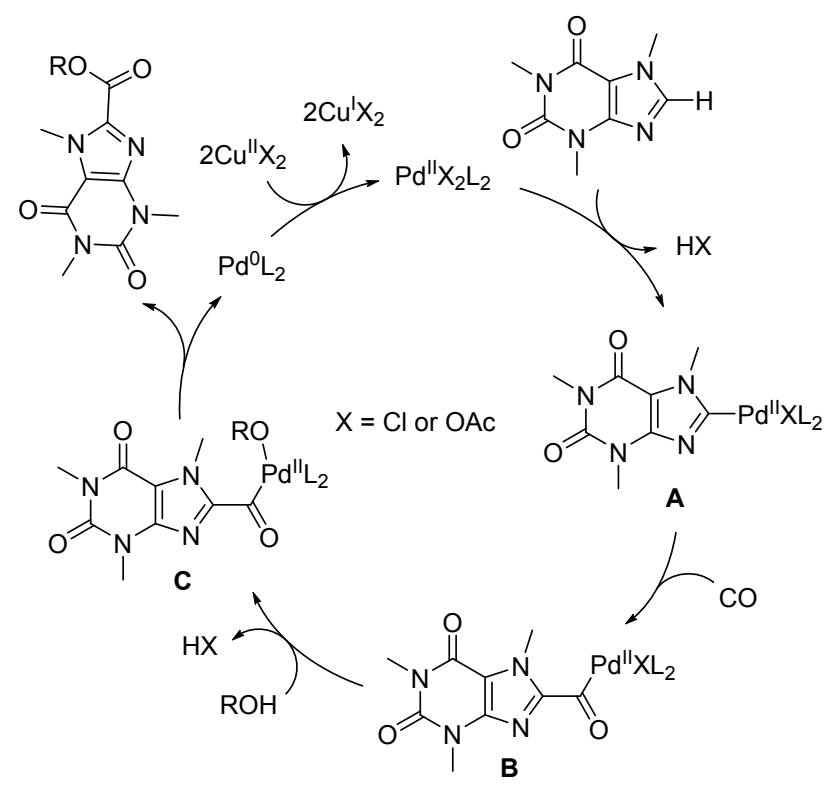

图式 1 可能的反应机理

Scheme 1 Proposed reaction mechanism

性导致羰基化反应不能进行, 中间体 $\mathbf{C}$ 可以经过还原消 除得到目标产物并释放出零价钯, 产生的零价钯经过二 价铜离子氧化后重新得到二价钯催化活性物种实现催 化循环。

\section{2 结论}

本实验采用 $\mathrm{PdCl}_{2}\left(\mathrm{PPh}_{3}\right)_{2}$ (10 mol\%)为催化剂, 以 $\mathrm{Cu}(\mathrm{OAc})_{2}$ (2.0 equiv.)作为氧化剂, 1,4 -二氧六环 $(1 \mathrm{~mL})$ 作 为反应溶剂, 在 $\mathrm{CO}(101 \mathrm{kPa})$ 气氛下, 温度为 $120{ }^{\circ} \mathrm{C}$, 反应时间为 $24 \mathrm{~h}$ 的反应体系下, 直接从咖啡因 $\mathrm{C}-\mathrm{H}$ 键 出发, 以 $\mathrm{CO}$ 作为 $\mathrm{C} 1$ 源, 与醇类物质发生直接氧化羰基 化反应合成 8 -酯基咖啡因衍生物. 该反应具有以下优 点: (1)反应无需对咖啡因原料预先官能化如生成卤代化 合物, 而是直接从咖啡因 $\mathrm{C}-\mathrm{H}$ 键出发一步合成 8-酯基 咖啡因衍生物; (2)反应以 $\mathrm{CO}$ 为碳源, 反应副产物为水, 具有高原子经济性, 符合现代绿色化学发展要求; (3)反 应无需高压操作, 所用 $\mathrm{CO}$ 为一个大气压，操作简便； (4)催化剂体系对底物的普适性强, 应用范围广, 可根据 实际需要设计合成各种 8-酯基咖啡因衍生物.

\section{3 实验部分}

\section{1 仪器与试剂}

${ }^{1} \mathrm{H}$ NMR 和 ${ }^{13} \mathrm{C}$ NMR 用 Bruker AM-400 (400 MHz) 型核磁共振仪器测定, TMS 为内标; HRMS 数据采用 Waters GCT Premier (EI-TOF) 仪器测定. 所有试剂分析 纯, 均为商业购买, 直接用于实验, 溶剂经过严格除水 干燥处理; 柱层析硅胶(200 300 目)为青岛海洋化工有
限公司生成, 购买后直接使用. 咖啡因底物按文献方法 合成 ${ }^{[14]}$.

\subsection{8 -酯基咖啡因衍生物的合成}

通用实验过程以咖啡因 $1 \mathrm{a}$ 与 $\mathrm{CO}$ 反应生成 $2 \mathrm{a}$ 为例: 室温下在连接有气球的 Schlenk 反应管中加入 $0.2 \mathrm{mmol}$ 咖啡因, $0.02 \mathrm{mmol}\left[\mathrm{PdCl}_{2}\left(\mathrm{PPh}_{3}\right)_{2}\right], 0.4 \mathrm{mmol} \mathrm{Cu}(\mathrm{OAc})_{2}$, 用 $\mathrm{CO}$ 气体抽换气三次. 用注射器注射 $1 \mathrm{~mL} 1,4$-二氧六 环和 $0.3 \mathrm{~mL}$ 试剂正丁醇, 然后将反应管放于 $120{ }^{\circ} \mathrm{C}$ 的 油浴锅中反应 $24 \mathrm{~h}$, 反应结束后冷却至室温后在通风樚 中缓慢释放 $\mathrm{CO}$ 气体. 浓缩混合物, 所得粗产物用石油 醚：乙酸乙酯的体积比为 $5: 1$ 的混合液体作为淋洗剂, 经柱层析得到最终产物 1,3,7-三甲基-2,6-二氧代-2,3,6,7四氢- $1 H$-嘌呤-8-甲酸丁酯(2a), 白色固体, 产率 $93 \% .{ }^{1} \mathrm{H}$ NMR (400 MHz, $\left.\mathrm{CDCl}_{3}\right) \delta: 4.42(\mathrm{t}, J=6.9 \mathrm{~Hz}, 2 \mathrm{H}), 4.35$ (s, 3H), $3.61(\mathrm{~s}, 3 \mathrm{H}), 3.41(\mathrm{~s}, 3 \mathrm{H}), 1.85 \sim 1.76(\mathrm{~m}, 2 \mathrm{H})$, $1.46(\mathrm{dd}, J=15.1,7.5 \mathrm{~Hz}, 2 \mathrm{H}), 0.97$ (t, $J=7.4 \mathrm{~Hz}, 3 \mathrm{H})$; ${ }^{13} \mathrm{C}$ NMR $\left(100 \mathrm{MHz}, \mathrm{CDCl}_{3}\right) \delta: 158.5,155.6,151.4,146.8$, $139.1,110.4,66.5,34.8,30.6,30.0,28.2,19.1,13.7$; HRMS calcd for $\mathrm{C}_{13} \mathrm{H}_{18} \mathrm{~N}_{4} \mathrm{O}_{4}: 294.1328$, found 294.1327.

1,3-二甲基-7-乙基-2,6-二氧代-2,3,6,7-四氢- $1 H$-嘌 呤-8-甲酸丁酯(2b): ${ }^{1} \mathrm{H}$ NMR (400 $\mathrm{MHz}, \mathrm{CDCl}_{3}$ ) $\delta: 4.87$ (q, $J=7.1 \mathrm{~Hz}, 2 \mathrm{H}), 4.42$ (t, $J=6.9 \mathrm{~Hz}, 2 \mathrm{H}), 3.61(\mathrm{~s}, 3 \mathrm{H})$, 3.42 (s, 3H), 1.80 (dd, $J=8.6,6.5 \mathrm{~Hz}, 2 \mathrm{H}), 1.45$ (q, $J=7.3$ $\mathrm{Hz}, 5 \mathrm{H}), 0.97$ (t, $J=7.4 \mathrm{~Hz}, 3 \mathrm{H}) ;{ }^{13} \mathrm{C}$ NMR $(100 \mathrm{MHz}$, $\left.\mathrm{CDCl}_{3}\right) \delta: 158.3,155.2,151.5,147.0,138.4,109.7,66.5$, 43.1, 30.5, 30.0, 28.2, 19.1, 16.5, 13.7; HRMS calcd for $\mathrm{C}_{14} \mathrm{H}_{20} \mathrm{~N}_{4} \mathrm{O}_{4}: 308.1485$, found 308.1487 .

1,3-二甲基-7-丙基-2,6-二氧代-2,3,6,7-四氢- $1 H$-嘌 呤-8-甲酸丁酯(2c): ${ }^{1} \mathrm{H} \mathrm{NMR}\left(400 \mathrm{MHz}, \mathrm{CDCl}_{3}\right.$ ) $\delta: 4.82$ $4.72(\mathrm{~m}, 2 \mathrm{H}), 4.41(\mathrm{t}, J=7.0 \mathrm{~Hz}, 2 \mathrm{H}), 3.61(\mathrm{~s}, 3 \mathrm{H}), 3.41(\mathrm{~s}$, $3 \mathrm{H}), 1.82(\mathrm{dd}, J=15.0,11.4,6.6 \mathrm{~Hz}, 4 \mathrm{H}), 1.45$ (dd, $J=$ $15.1,7.5 \mathrm{~Hz}, 2 \mathrm{H}), 0.96(\mathrm{td}, J=7.4,6.4 \mathrm{~Hz}, 6 \mathrm{H}) ;{ }^{13} \mathrm{C} \mathrm{NMR}$ $\left(100 \mathrm{MHz}, \mathrm{CDCl}_{3}\right) \delta: 158.4,155.3,151.5,147.0,138.7$, $110.0,66.5,48.9,30.5,30.0,28.2,24.7,19.1,13.7,10.7$; HRMS calcd for $\mathrm{C}_{15} \mathrm{H}_{22} \mathrm{~N}_{4} \mathrm{O}_{4}: 322.1641$, found 322.1640 .

1,3-二甲基-7-(3-溴丙基)-2,6-二氧代-2,3,6,7-四氢$1 H$-嘌呤-8-甲酸丁酯(2d): ${ }^{1} \mathrm{H} \mathrm{NMR}\left(400 \mathrm{MHz}, \mathrm{CDCl}_{3}\right) \delta$ : $5.01 \sim 4.89(\mathrm{~m}, 2 \mathrm{H}), 4.43(\mathrm{t}, J=7.0 \mathrm{~Hz}, 2 \mathrm{H}), 3.61(\mathrm{~s}, 3 \mathrm{H})$, $3.50 \sim 3.35(\mathrm{~m}, 5 \mathrm{H}), 2.49 \sim 2.37(\mathrm{~m}, 2 \mathrm{H}), 1.81$ (dd, $J=8.5$, $6.5 \mathrm{~Hz}, 2 \mathrm{H}), 1.46$ (dd, $J=15.1,7.5 \mathrm{~Hz}, 2 \mathrm{H}), 0.97$ (t, $J=7.4$ $\mathrm{Hz}, 3 \mathrm{H}) ;{ }^{13} \mathrm{C}$ NMR $\left(100 \mathrm{MHz}, \mathrm{CDCl}_{3}\right) \delta: 158.4,155.3$, $151.4,147.1,138.8,109.9,66.7,46.6,33.7,30.5,30.1$, 29.0, 28.3, 19.1, 13.7; HRMS calcd for $\mathrm{C}_{15} \mathrm{H}_{21} \mathrm{BrN}_{4} \mathrm{O}_{4}$ : 400.0746, found 400.0746 .

1,3-二甲基-7-芐基-2,6-二氧代-2,3,6,7-四氢- $1 H$-嘌 
呤-8-甲酸丁酯(2e): ${ }^{1} \mathrm{H} \mathrm{NMR}\left(400 \mathrm{MHz}, \mathrm{CDCl}_{3}\right) \delta:$ 7.33 7.25 (m, 5H), 6.09 (s, 2H), 4.40 (t, $J=6.9 \mathrm{~Hz}, 2 \mathrm{H}), 3.63$ (s, $3 \mathrm{H}), 3.42(\mathrm{~s}, 3 \mathrm{H}), 1.81 \sim 1.70(\mathrm{~m}, 2 \mathrm{H}), 1.40$ (dd, $J=15.1$, $7.5 \mathrm{~Hz}, 2 \mathrm{H}), 0.95(\mathrm{t}, J=7.4 \mathrm{~Hz}, 3 \mathrm{H}) ;{ }^{13} \mathrm{C}$ NMR $(100 \mathrm{MHz}$, $\left.\mathrm{CDCl}_{3}\right) \delta: 158.5,155.4,151.4,147.1,138.8,136.3,128.7$, 128.1, 127.5, 109.9, 66.5, 50.0, 30.5, 30.0, 28.3, 19.0, 13.7; HRMS calcd for $\mathrm{C}_{19} \mathrm{H}_{22} \mathrm{~N}_{4} \mathrm{O}_{4}: 370.1641$, found 370.1640 .

3,7-二甲基-1-乙基-2,6-二氧代-2,3,6,7-四氢- $1 H$-嘌 呤-8-甲酸丁酯(2f): ${ }^{1} \mathrm{H} \mathrm{NMR}\left(400 \mathrm{MHz}, \mathrm{CDCl}_{3}\right) \delta: 4.42$ (t, $J=6.9 \mathrm{~Hz}, 2 \mathrm{H}), 4.34$ (s, 3H), 4.08 (q, $J=7.1 \mathrm{~Hz}, 2 \mathrm{H}), 3.60$ (s, 3H), 1.80 (dd, $J=8.5,6.5 \mathrm{~Hz}, 2 \mathrm{H}), 1.45$ (dd, $J=15.1$, $7.5 \mathrm{~Hz}, 2 \mathrm{H}), 1.24$ (t, $J=7.1 \mathrm{~Hz}, 3 \mathrm{H}), 0.97$ (t, $J=7.4 \mathrm{~Hz}$, $3 \mathrm{H}) ;{ }^{13} \mathrm{C}$ NMR $\left(100 \mathrm{MHz}, \mathrm{CDCl}_{3}\right) \delta: 158.5,155.3,151.0$, 146.9, 139.0, 110.5, 66.4, 36.8, 34.7, 30.5, 29.9, 19.1, 13.7, 13.2; HRMS calcd for $\mathrm{C}_{14} \mathrm{H}_{20} \mathrm{~N}_{4} \mathrm{O}_{4}: 308.1485$, found 308.1482 .

3,7-二甲基-1-丙基-2,6-二氧代-2,3,6,7-四氢- $1 H$-嘌 呤-8-甲酸丁酯(2g): ${ }^{1} \mathrm{H}$ NMR (400 $\left.\mathrm{MHz}, \mathrm{CDCl}_{3}\right) \delta: 4.42$ (t, $J=6.9 \mathrm{~Hz}, 2 \mathrm{H}), 4.34(\mathrm{~s}, 3 \mathrm{H}), 4.02 \sim 3.90(\mathrm{~m}, 2 \mathrm{H}), 3.60$ (s, 3H), $1.86 \sim 1.74(\mathrm{~m}, 2 \mathrm{H}), 1.68(\mathrm{t}, J=7.6 \mathrm{~Hz}, 3 \mathrm{H}), 1.45$ (dd, $J=15.1,7.5 \mathrm{~Hz}, 2 \mathrm{H}), 0.97(\mathrm{t}, J=7.3 \mathrm{~Hz}, 5 \mathrm{H}) ;{ }^{13} \mathrm{C}$ NMR $\left(100 \mathrm{MHz}, \mathrm{CDCl}_{3}\right) \delta: 158.5,155.5,151.2,146.9$, 139.1, 110.5, 66.4, 43.2, 34.7, 30.5, 29.9, 21.2, 19.1, 13.7, 11.3; HRMS calcd for $\mathrm{C}_{15} \mathrm{H}_{22} \mathrm{~N}_{4} \mathrm{O}_{4}$ : 322.1641, found 322.1640 .

3,7-二甲基-1-烯丙基-2,6-二氧代-2,3,6,7-四氢- $1 H$ 嘌呤-8-甲酸丁酯(2h): ${ }^{1} \mathrm{H}$ NMR $\left(400 \mathrm{MHz}, \mathrm{CDCl}_{3}\right) \delta$ : $5.89(\mathrm{dd}, J=8.8,8.3 \mathrm{~Hz}, 1 \mathrm{H}), 5.31 \sim 5.14(\mathrm{~m}, 2 \mathrm{H}), 4.62(\mathrm{~d}$, $J=5.8 \mathrm{~Hz}, 2 \mathrm{H}), 4.42(\mathrm{t}, J=6.9 \mathrm{~Hz}, 2 \mathrm{H}), 4.34(\mathrm{~s}, 3 \mathrm{H}), 3.61$ (s, 3H), $1.87 \sim 1.74(\mathrm{~m}, 2 \mathrm{H}), 1.45(\mathrm{dd}, J=15.1,7.5 \mathrm{~Hz}$, 2H), 0.97 (t, $J=7.4 \mathrm{~Hz}, 3 \mathrm{H}) ;{ }^{13} \mathrm{C}$ NMR (100 MHz, $\mathrm{CDCl}_{3}$ ) $\delta: 158.5,155.2,151.0,147.0,139.2,131.9,117.9,110.4$, 66.5, 43.6, 34.8, 30.5, 30.0, 19.1, 13.7; HRMS calcd for $\mathrm{C}_{15} \mathrm{H}_{20} \mathrm{~N}_{4} \mathrm{O}_{4}: 320.1485$, found 320.1486 .

1,3-二甲基-7-烯丙基-2,6-二氧代-2,3,6,7-四氢- $1 H$ 嘌呤-8-甲酸丁酯(2i): ${ }^{1} \mathrm{H}$ NMR (400 MHz, $\mathrm{CDCl}_{3}$ ) $\delta: 7.03$ (d, $J=15.6 \mathrm{~Hz}, 1 \mathrm{H}), 5.65(\mathrm{dd}, J=5.3,1.7 \mathrm{~Hz}, 2 \mathrm{H}), 4.41$ (t, $J=6.9 \mathrm{~Hz}, 2 \mathrm{H}), 4.10$ (t, $J=6.7 \mathrm{~Hz}, 2 \mathrm{H}), 3.63$ (s, 3H), 3.41 (s, 3H), 1.79 (dd, $J=8.6,6.4 \mathrm{~Hz}, 2 \mathrm{H}), 1.64 \sim 1.56(\mathrm{~m}, 2 \mathrm{H})$, $1.00 \sim 0.94(\mathrm{~m}, 3 \mathrm{H}) ;{ }^{13} \mathrm{C}$ NMR $\left(100 \mathrm{MHz}, \mathrm{CDCl}_{3}\right) \delta$ : $155.0,151.5,147.6,141.7,139.0,132.9,118.4,108.4$, 64.7, 49.2, 29.8, 28.2, 19.1, 13.7; HRMS calcd for $\mathrm{C}_{15} \mathrm{H}_{20} \mathrm{~N}_{4} \mathrm{O}_{4}: 320.1485$, found 320.1483 .

1,3,7-三甲基-2,6-二氧代-2,3,6,7-四氢- $1 H$-嘌呤-8-甲 酸-3-丁烯酯(2j): ${ }^{1} \mathrm{H}$ NMR (400 MHz, $\left.\mathrm{CDCl}_{3}\right) \delta: 5.83$ (dd,
$J=17.1,10.3 \mathrm{~Hz}, 1 \mathrm{H}), 5.23 \sim 5.07(\mathrm{~m}, 2 \mathrm{H}), 4.47$ (t, $J=6.9$ $\mathrm{Hz}, 2 \mathrm{H}), 4.34$ (s, 3H), 3.60 (s, 3H), 3.40 (s, 3H), 2.57 (q, $J=6.9 \mathrm{~Hz}, 2 \mathrm{H}) ;{ }^{13} \mathrm{C}$ NMR $\left(100 \mathrm{MHz}, \mathrm{CDCl}_{3}\right) \delta: 158.3$, 133.1, 118.0, 65.4, 34.7, 32.9, 30.0, 28.2; HRMS calcd for $\mathrm{C}_{13} \mathrm{H}_{16} \mathrm{~N}_{4} \mathrm{O}_{4}: 292.1172$, found 292.1170 .

1,3,7-三甲基-2,6-二氧代-2,3,6,7-四氢- $1 H$-嘌呤-8-甲 酸芐酯(2k): ${ }^{1} \mathrm{H}$ NMR (400 MHz, $\left.\mathrm{CDCl}_{3}\right) \delta: 7.53 \sim 7.32$ (m, 5H), 5.45 (s, 2H), 4.34 (s, 3H), $3.61(\mathrm{~s}, 3 \mathrm{H}), 3.41(\mathrm{~s}$, $3 \mathrm{H}) ;{ }^{13} \mathrm{C}$ NMR $\left(100 \mathrm{MHz}, \mathrm{CDCl}_{3}\right) \delta: 158.3,155.6,151.4$, $146.8,138.8,134.8,129.7 \sim 128.6,110.5,68.0,34.8,30.0$, 28.2; HRMS calcd for $\mathrm{C}_{16} \mathrm{H}_{16} \mathrm{~N}_{4} \mathrm{O}_{4}$ : 328.1172, found 328.1170 .

1,3,7-三甲基-2,6-二氧代- $2,3,6,7$-四氢- $1 H$-嘌呤-8-甲 酸甲酯(2I): ${ }^{1} \mathrm{H}$ NMR (400 MHz, $\left.\mathrm{CDCl}_{3}\right) \delta: 4.36$ (s, 3H), $4.01(\mathrm{~s}, 3 \mathrm{H}), 3.60(\mathrm{~s}, 3 \mathrm{H}), 3.41(\mathrm{~s}, 3 \mathrm{H}) ;{ }^{13} \mathrm{C}$ NMR $(100$ $\left.\mathrm{MHz}, \mathrm{CDCl}_{3}\right) \delta: 158.8,155.5,151.4,146.8,138.6,110.6$, 53.2, 34.7, 30.0, 28.2; HRMS calcd for $\mathrm{C}_{10} \mathrm{H}_{12} \mathrm{~N}_{4} \mathrm{O}_{4}$ : 252.0859 , found 252.0860 .

1,3,7-三甲基-2,6-二氧代-2,3,6,7-四氢- $1 H$-嘌呤-8-甲 酸乙酯(2m): ${ }^{1} \mathrm{H}$ NMR (400 MHz, $\mathrm{CDCl}_{3}$ ) $\delta: 4.48$ (q, $J=$ $7.1 \mathrm{~Hz}, 2 \mathrm{H}), 4.35$ (s, 3H), 3.61 (s, 3H), 3.41 (s, 3H), 1.45 (t, $J=7.1 \mathrm{~Hz}, 3 \mathrm{H}) ;{ }^{13} \mathrm{C}$ NMR $\left(100 \mathrm{MHz}, \mathrm{CDCl}_{3}\right) \delta: 158.3$, 155.6, 151.4, 146.8, 139.0, 110.4, 65.8, 34.8, 33.0, 30.0, 28.2; HRMS calcd for $\mathrm{C}_{11} \mathrm{H}_{14} \mathrm{~N}_{4} \mathrm{O}_{4}$ : 266.1015, found 266.1015 .

1,3,7-三甲基-2,6-二氧代-2,3,6,7-四氢- $1 H$-嘌呤-8-甲 酸异丙酯(2n): ${ }^{1} \mathrm{H}$ NMR (400 MHz, $\left.\mathrm{CDCl}_{3}\right) \delta: 5.36 \sim 5.24$ (m, $1 \mathrm{H}), 4.33(\mathrm{~s}, 3 \mathrm{H}), 3.60(\mathrm{~s}, 3 \mathrm{H}), 3.46 \sim 3.35(\mathrm{~m}, 3 \mathrm{H})$, $1.43(\mathrm{~d}, J=6.3 \mathrm{~Hz}, 6 \mathrm{H}) ;{ }^{13} \mathrm{C} \mathrm{NMR}\left(100 \mathrm{MHz}, \mathrm{CDCl}_{3}\right) \delta$ : 158.0, 155.6, 151.4, 146.8, 139.5, 110.3, 70.9, 34.7, 30.0, 28.2, 21.7; HRMS calcd for $\mathrm{C}_{12} \mathrm{H}_{16} \mathrm{~N}_{4} \mathrm{O}_{4}: 280.1172$, found 280.1175 .

1,3,7-三甲基-2,6-二氧代-2,3,6,7-四氢- $1 H$-嘌呤-8-甲 酸-3-甲氧基芐酯(2p): ${ }^{1} \mathrm{H}$ NMR (400 $\left.\mathrm{MHz}, \mathrm{CDCl}_{3}\right) \delta$ : $7.29(\mathrm{t}, J=7.9 \mathrm{~Hz}, 1 \mathrm{H}), 7.06 \sim 7.02(\mathrm{~m}, 1 \mathrm{H}), 7.00(\mathrm{~s}, 1 \mathrm{H})$, $6.90 \sim 6.86(\mathrm{~m}, 1 \mathrm{H}), 5.41(\mathrm{~s}, 2 \mathrm{H}), 4.33(\mathrm{~d}, J=0.9 \mathrm{~Hz}, 3 \mathrm{H})$, 3.80 (d, $J=0.8 \mathrm{~Hz}, 3 \mathrm{H}), 3.59$ (d, $J=1.0 \mathrm{~Hz}, 3 \mathrm{H}), 3.40$ (d, $J=1.0 \mathrm{~Hz}, 3 \mathrm{H}) ;{ }^{13} \mathrm{C}$ NMR $\left(100 \mathrm{MHz}, \mathrm{CDCl}_{3}\right) \delta: 159.8$, $158.3,155.6,151.4,146.8,138.8,136.3,129.8,120.8$, $114.2,110.5,67.8,55.3,34.8,30.0,28.2$; HRMS calcd for $\mathrm{C}_{17} \mathrm{H}_{18} \mathrm{~N}_{4} \mathrm{O}_{4}: 358.1277$, found 358.1277 .

辅助材料(Supporting Information) 所有化合物的 ${ }^{1} \mathrm{H}$ NMR 和 ${ }^{13} \mathrm{C}$ NMR 谱图. 这些材料可以免费从本刊网站 (http://sioc-journal.cn/)上下载. 


\section{References}

[1] (a) Quintero-Duque, S.; Dyballa, K. M.; Fleischer, I. Tetrahedron Lett. 2015, 56, 2634.

(b) Wu, X.-F.; Fang, X.; Wu, L.; Jackstell, R.; Neumann, H.; Beller, M. Acc. Chem. Res. 2014, 47, 1041.

(c) Sumino, S.; Fusano, A.; Fukuyama, T.; Ryu, I. Acc. Chem. Res. 2014, 47, 1563.

(d) Barnard, C. F. J. Organometallics 2008, 27, 5402.

(e) Wu, X.-F.; Neumann, H.; Beller, M. Chem. Rev. 2013, 113, 1.

(f) Omae, I. Coordin. Chem. Rev. 2011, 255, 139.

[2] (a) Schoenberg, A.; Bartoletti, I.; Heck, R. F. J. Org. Chem. 1974, 39,3318 .

(b) Schoenberg, A.; Heck, R. F. J. Org. Chem. 1974, 39, 3327.

(c) Schoenberg, A.; Heck, R. F. J. Am. Chem. Soc. 1974, 96, 7761.

[3] (a) Wu, L.; Fang, X.; Liu, Q.; Jackstell, R.; Beller, M.; Wu, X.-F. ACS Catal. 2014, 4, 2977.

(b) Sumino, S.; Fusano, A.; Fukuyama, T.; Ryu, I. Acc. Chem. Res. 2014, 47, 1563.

(c) Wu, X.-F.; Neumann, H.; Beller, M. Chem. Soc. Rev. 2011, 40, 4986.

(d) Brennführer, A.; Neumann, H.; Beller, M. Angew. Chem., Int. Ed. 2009, 48, 4114.

(e) Sargent, B. T.; Alexanian, E. J. J. Am. Chem. Soc. 2016, 138, 7520 .

[4] (a) Liu, B.; Hu, F.; Shi, B.-F. ACS Catal. 2015, 5, 1863.

(b) Wu, X.-F.; Neumann, H.; Beller, M. ChemSusChem 2013, 6, 229.

(c) Liu, Q.; Zhang, H.; Lei, A. Angew. Chem., Int. Ed. 2011, 50, 10788.

(d) Gadge, S. T.; Bhanage, B. M. RSC Adv. 2014, 4, 10367.

(e) Fenner, S.; Ackermann, L. Green Chem. 2016, 18, 3804.

(f) Boogaerts, I. I. F.; Nolan, S. P. J. Am. Chem. Soc. 2010, 132, 8858 .

[5] (a) Müller, C. E.; Jacobson, K. A. Biochim. Biophys. Acta 2011, 1808,1290

(b) Stydom, B.; Bergh, J. J.; Petzer, J. P. Eur. J. Med. Chem. 2011, 46, 3474.

(c) Van der Walt, M. M.; Terre'Blanche, G.; Petzer, A.; Lourens, A. C. U.; Petzer, J. P. Bioorg. Chem. 2013, 49, 49.

(d) Rivara, S.; Piersanti, G.; Bartoccini, F.; Diamantini, G.; Pala, D.; Riccioni, T.; Stasi, M. A.; Cabri, W.; Borsini, F.; Mor, M.; Tarzia, G.; Minetti, P. J. Med.Chem. 2013, 56, 1247.

(e) Baraldi, P. G.; Baraldi, S.; Saponaro, G.; Preti, D.; Romagnoli, R.; Piccagli, L.; Cavalli, A.; Recanatini, M.; Moorman, A. R.; Zaid, A. N.; Varani, K.; Borea, P. A.; Tabrizi, M. J. J. Med. Chem. 2012, $55,797$.

[6] Uchil, V.; Seo, B.; Nair, V. J. Org. Chem. 2007, 72, 8577.
[7] (a) Zhang, H.; Zhou, L.; Zhu, Z.; Yang, C. Chem. Eur. J. 2016, 22, 9886.

(b) Kocaoglu, O.; Carlson, E. E. Nat. Chem. Biol. 2016, 12, 472.

(c) Rice, D. R.; Clear, K. J.; Smith, B. D. Chem. Commun. 2016, $52,8787$.

[8] (a) Greco, N. J.; Tor, Y. Tetrahedron 2007, 63, 3515.

(b) Kim, D.; Lee, J. H.; Hong, S.-S.; Hong, S. Org. Lett. 2010, 12, 1212.

(c) Zhao, D.; Wang, W.; Yang, F.; Lan, J.; Yang, L.; Gao, G.; You, J. Angew. Chem., Int. Ed. 2009, 48, 3296.

(d) Huang, Y.; Song, F.; Wang, Z.; Xi, P.; Wu, N.; Wang, Z.; Lan, J.; You, J. Chem. Commun. 2012, 48, 2864.

[9] Heer, J. P.; Smith, I. E. D. WO 2007017265, 2007 [Chem. Abstr. 2007, 146, 251660].

[10] (a) Neufeldt, S. R.; Sanford, M. S. Acc. Chem. Res. 2012, 45, 936. (b) Li, H.; Shi, Z. Prog. Chem. 2010, 22, 1414 (in Chinese). (李湖, 施章杰, 化学进展, 2010, 22, 1414).

(c) Lyons, T. W.; Sanford, M. S. Chem. Rev. 2010, 110, 1147.

(d) Liu, C.; Yuan, J.; Gao, M.; Tang, S.; Li, W.; Shi, R.; Lei, A. Chem. Rev. 2015, 115, 12138.

(e) Chen, Z.; Wang, B.; Zhang, J.; Yu, W.; Liu, Z.; Zhang, Y. Org. Chem. Front. 2015, 2, 1107.

(f) Gang, F.; Xu, G.; Dong, T.; Yang, L.; Du, Z. Chin. J. Org. Chem. 2015, 35, 1428 (in Chinese).

(刚芳莉, 徐关利, 董涛生, 杨丽, 杜正银, 有机化学, 2015, 35, 1428.)

[11] (a) Lang, R.; Shi, L.; Li, D.; Xia, C.; Li, F. Org. Lett. 2012, 14, 4130.

(b) Chen, M.; Ren, Z.-H.; Wang, Y.-Y.; Guan, Z.-H. J. Org. Chem. 2015, 80, 1258.

(c) Li, X.; Li, X.; Jiao, N. J. Am. Chem. Soc. 2015, 137, 9246.

(d) Yoo, E. J.; Wasa, M, Yu, J.-Q. J. Am. Chem. Soc. 2010, 132, 17378 .

(e) Lian, Z.; Friis, S. D.; Skrydstrup, T. Chem. Commun. 2015, 51, 1870.

(f) Liu, B.; Hu, F.; Shi, B.-F. ACS Catal. 2015, 5, 1863.

[12] Chen, M.; Ren, Z.-H.; Wang, R.-Y.; Guan, Z.-H. Angew. Chem., Int. Ed. 2013, 52, 14196.

[13] Lang, R.; Wu, J.; Shi, L.; Xia, C.; Li, F. Chem. Commun. 2011, 47. 12553.

[14] Zhang, H.; Liu, D.; Chen, C.; Liu, C.; Lei, A. Chem. Eur. J. 2011, $17,9581$.

[15] (a) Malakar, C. C.; Schmidt, D.; Conrad, J.; Beifuss, U. Org. Lett. 2011, 13, 1378.

(b) Daly, J. W.; Padgett, W. L.; Shamim, M. T. J. Med. Chem. 1986, 29, 1305.

(Li, L.; Fan, Y.) 